\title{
Expression of a Truncated Form of Ribosomal Protein L3 Confers Resistance to Pokeweed Antiviral Protein and the Fusarium Mycotoxin Deoxynivalenol
}

\author{
Rong Di and Nilgun E. Tumer \\ Biotechnology Center for Agriculture and the Environment and the Department of Plant Biology and Pathology, \\ Cook College, Rutgers University, New Brunswick, New Jersey 08901-8520, U.S.A.
}

Submitted 1 February 2005. Accepted 24 March 2005.

\begin{abstract}
The contamination of important agricultural products such as wheat, barley, or maize with the trichothecene mycotoxin deoxynivalenol (DON) due to infection with Fusarium species is a worldwide problem. Trichothecenes inhibit protein synthesis by targeting ribosomal protein L3. Pokeweed antiviral protein (PAP), a ribosome-inactivating protein binds to $\mathrm{L3}$ to depurinate the $\alpha$-sarcin/loop of the large rRNA. Plants transformed with the wild-type PAP show lesions and express very low levels of PAP because PAP autoregulates its expression by destabilizing its own mRNA. We show here that transgenic tobacco plants expressing both the wild-type PAP and a truncated form of yeast L3 (L3 $\Delta$ ) are phenotypically normal. PAP mRNA and protein levels are very high in these plants, indicating that L3 $\Delta$ suppresses the autoregulation of PAP mRNA expression. Ribosomes are not depurinated in the transgenic plants expressing PAP and $L 3 \Delta$, even though PAP is associated with ribosomes. The expression of the endogenous tobacco ribosomal protein $\mathrm{L} 3$ is up-regulated in these plants and they are resistant to the Fusarium mycotoxin DON. These results demonstrate that expression of an $\mathrm{N}$-terminal fragment of yeast $\mathrm{L3}$ leads to trans-dominant resistance to PAP and the trichothecene mycotoxin DON, providing evidence that both toxins target $\mathrm{L3}$ by a common mechanism.
\end{abstract}

Additional keywords: Fusarium head blight, translation inhibitor.

The trichothecenes are a family of low-molecular-weight sesquiterpenoid mycotoxins synthesized by various Fusarium species. The contamination of important agricultural products, such as wheat, barley, or maize with the trichothecene mycotoxin deoxynivalenol (DON) due to infection with Fusarium graminearum and Fusarium culmorum is a worldwide problem because DON accumulates in grain to levels posing a threat to human and animal health (McLaughlin et al. 1977). Favorable weather conditions led to a dramatic reemergence of Fusarium head blight (FHB) in the United States in the last decade (McMullen et al. 1997), resulting in economic losses of $\$ 2.7$ billion in small grain cereals (Nganje et al. 2001). Trichothecene mycotoxins interact with the peptidyltransferase site of eukaryotic ribosomes and inhibit protein synthesis (Feinberg and McLaughlin 1989). In addition, they cause membrane

Corresponding author: N. E. Tumer; Telephone: +1.732 .932 .8165 ext. 215; Fax: +1.732.932.6535; E-mail: tumer@aesop.rutgers.edu damage (Feinberg and McLaughlin 1989; Khachatourians 1990). Trichothecene-resistant yeast mutants contain alterations in the ribosomal protein L3, encoded by RPL3. A mutation in the RPL3 gene that conferred resistance to the trichothecene trichodermin was initially identified in yeast (Grant et al. 1976; Fernandez-Lobato et al. 1990; Fried and Warner 1981; Jimenez et al. 1975; Schindler et al. 1974) and was referred to as tcm-1 (Fried and Warner 1981). Further studies provided evidence that DON has the same mode of action as the other trichothecenes (Fernandez-Lobato et al. 1990). Gene disruption mutants of Fusarium graminearum that have lost the ability to produce trichothecenes are less virulent on their hosts, providing evidence that trichothecenes act as virulence factors (McCormick 2003; Proctor et al. 1995). Thus, resistance to DON may be a critical component of resistance to Fusarium infection because Fusarium strains will most likely be less virulent on plants that are resistant to DON, resulting in a reduction in DON accumulation.

Ribosome reconstitution experiments demonstrated that L3 is one of the first ribosomal proteins to be assembled into the ribosome and it is one of only three proteins required for peptidyltransferase activity (Hampl et al. 1981; Roth and Nierhaus 1980). The L3 binding site on the ribosome has been localized to the $\alpha$-sarcin/ricin loop (SRL) in Escherichia coli $23 \mathrm{~S}$ rRNA (Uchiumi et al. 1999). The SRL is a highly conserved stemloop structure in the large rRNA, which interacts with the elongation factors during protein synthesis (Wool et al. 2000). Pokeweed antiviral protein (PAP), a 29-kDa ribosome inactivating protein (RIP) isolated from pokeweed plants also binds to the SRL and catalytically removes specific purine residues, inhibiting translation (Parikh and Tumer 2004). We have shown that PAP depurinates the SRL by binding to L3. The mak8-1 allele of $R P L 3$, which confers resistance to trichodermin in yeast, also confers resistance to PAP because PAP does not bind to mak8-1 ribosomes (Hudak et al. 1999). Since the mutations in L3 that confer resistance to trichodermin also confer resistance to PAP, trichothecene mycotoxins and PAP may bind to the same site on L3. We therefore postulated that introduction of a modified form of L3 may prevent translation inhibition by DON and may also protect ribosomes from depurination by PAP.

Since RIPs such as PAP and ricin are quite stable, one or a few molecules are sufficient to kill cells (Olsnes and Sandvig 1988). Due to their cytotoxicity to dividing cells, PAP and ricin have been used as the cytotoxic component of immunotoxins directed against cancer cell targets (Bolognesi and Polito 2004). Wide availability and the ease of production of 
RIPs such as ricin resulted in their development as agents of bioterrorism and biological warfare (Christopher et al. 1997; Henghold 2004; Zapor and Fishbain 2004). Currently, there are no antidotes that can protect people exposed to RIPs. The development of effective inhibitors has been impeded by an insufficient understanding of the relationship between their ribosome interactions and cytotoxicity. We show here that coexpression of an N-terminal fragment of yeast L3 encoding the first 99 amino acids (L3 $\Delta$ ) together with the wild-type PAP in transgenic tobacco plants eliminates the toxicity of PAP, its ability to destabilize its own mRNA and depurinate ribosomes. The endogenous tobacco L3 levels are higher in plants expressing the yeast $\mathrm{L} 3 \Delta$, and they are resistant to the trichothecene mycotoxin DON. This is the first demonstration that introduction of a modified target of a RIP and a trichothecene mycotoxin into transgenic plants leads to trans-dominant resistance to both toxins, providing evidence that they target L3 through a common mechanism.

\section{RESULTS}

\section{Expression of L3 and PAP genes}

in transgenic tobacco plants.

To determine if increased levels of L3 in transgenic plants would confer resistance to DON, we have transformed tobacco plants with the full-length L3 gene from yeast, as well as a truncated form of L3 (L3 $\Delta$ ) that contains only the first 99 amino acids (Fig. 1A). The yeast L3 $\Delta$ was included in these studies because our previous results have shown that a doublestranded RNA construct corresponding to the C-terminal truncated form of the tobacco RPL3A gene down-regulated the $R P L 3 A$ mRNA and up-regulated the RPL3B mRNA and L3 protein levels in transgenic tobacco plants (Popescu and Tumer 2004). The full-length yeast L3 (NT250) and L3 $\Delta$ (NT252) were transformed into tobacco plants (N. tabacum cv. Samsun $\mathrm{NN}$ ) either alone or together with PAP. Twenty transgenic lines were generated with each construct encoding the full-length L3 (NT250) or the L3 $\Delta$ (NT252). These plants were pheno- typically normal and indistinguishable from the wild-type plants based on their appearance and growth characteristics (data not shown).

A total of 12 neomycin phosphotransferase (NPTII) positive transgenic tobacco lines containing the full-length L3 and wild-type PAP (NT243) were identified. The transformation frequency, defined as the number of transgenic plants obtained per initial leaf disk times 100 , was approximately $24 \%$, which was considerably greater than the transformation frequency $(0.7 \%)$ previously observed when wild-type PAP was transformed into tobacco (Lodge et al. 1993). PAP mRNA and protein levels were very low in transgenic plants expressing the wild-type PAP or an enzymatically active variant, PAPv (Lodge et al. 1993; Zoubenko et al. 2000), because PAP autoregulates its expression by destabilizing its own mRNA (Parikh et al. 2002). Immunoblot analysis of the primary transformants, containing L3+PAP (NT243) indicated different levels of PAP expression (Fig. 1B). Lines 243-7 and 243-9 had the highest level of PAP. These plants showed lesions on their leaves, as previously reported for transgenic plants expressing only the wild-type PAP or the enzymatically active variant, PAPv (Lodge et al. 1993) and did not produce viable seeds. The PAP protein level in lines 243-6 and 243-8 was much lower compared with that in 243-7 and 243-9 (Fig. 1B), and these lines were fertile. As shown in Figure 2, lesion severity on the leaves of the homozygous progeny from lines 243-6 and 243-8 was much less compared with that of lesions observed on plants expressing PAPv.

A total of 20 NPTII positive transgenic tobacco lines containing the wild-type PAP and L3 $\Delta$ (NT245) were identified. All lines containing PAP+L $3 \Delta$ were fertile. Homozygous progeny of transgenic lines $245-1$ and $245-2$ were phenotypically normal and indistinguishable from the wild-type plants (Fig. 2). Immunoblot analysis indicated that each transgenic line containing NT245 expressed a high level of PAP (Fig. 1B). These results demonstrated that expression of L $3 \Delta$ eliminated the cytotoxicity of PAP and increased the PAP protein levels in the transgenic plants.

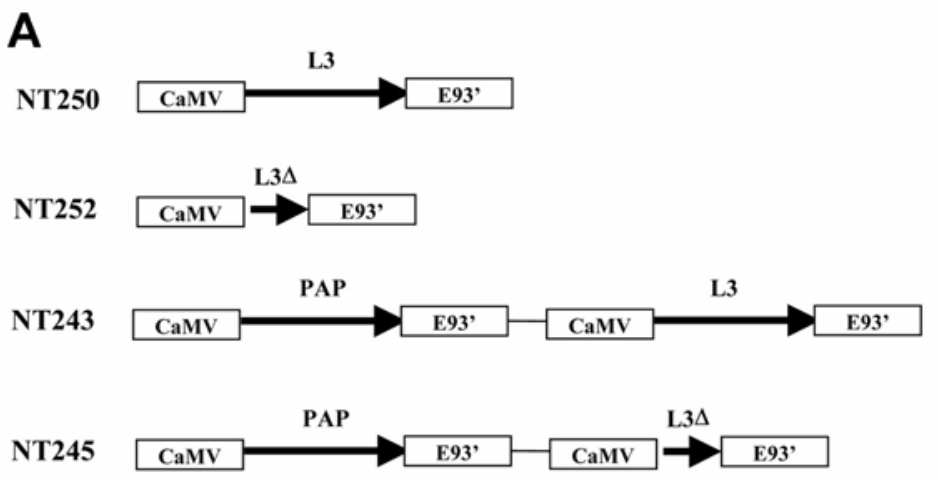

B

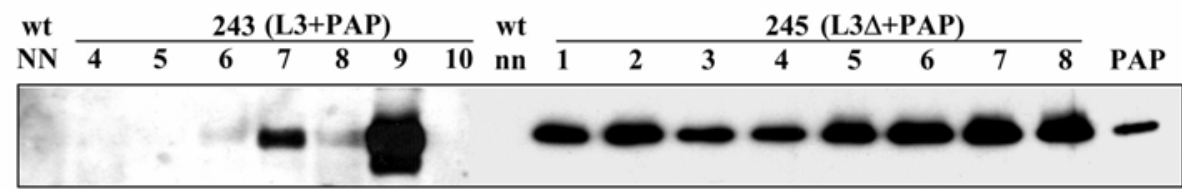

Fig. 1. Constructs used to generate transgenic tobacco plants and analysis of their expression. A, Yeast L3, L3 $\Delta$, and pokeweed antiviral protein (PAP) genes were cloned downstream of the enhanced $35 \mathrm{~S}$ promoter from Cauliflower mosaic virus (CaMV) and contained the pea small subunit of the ribulose-1,5bisphosphate carboxylase E9 polyadenylation sequences at their $3^{\prime}$ end. B, Immunoblot analysis of PAP expression in the primary transgenic tobacco lines. Total protein $(10 \mu \mathrm{g})$ from each transgenic and wild-type control line (wt NN and wt nn) was separated on a $10 \%$ sodium dodecyl sulfate-polyacrylamide gel electrophoresis and was subjected to immunoblot analysis using polyclonal anti-PAP immunoglobulin G (1:2,000). Purified PAP from pokeweed (10 ng) was used as the standard. The numbers above each lane refer to the numbers of the transgenic lines. 


\section{Steady state levels of PAP and L3 mRNA} in transgenic plants.

Real-time polymerase chain reaction (PCR) analysis was used to analyze expression of the full-length yeast L3 and L3 $\Delta$ in the homozygous progeny of the transgenic lines, using a set of specific primers designed for the N-terminal amino acids +22 to +48 (Fig. 3A, nucleotides +67 to +145 ), which are highly divergent among the different ribosomal protein L3 genes. These primers did not hybridize to the endogenous tobacco $R P L 3 A$ or $R P L 3 B$ genes, indicating that they were specific for the yeast RPL3 gene (data not shown). The mRNA levels were normalized against tobacco tubulin A1 mRNA as an internal control. Real-time PCR analysis was performed at least three times, using two to three replicates per sample. As shown in Figure $3 \mathrm{~A}, \mathrm{~L} 3 \Delta \mathrm{mRNA}$ was expressed at 50 - to 2,000-fold higher levels in plants containing L3 $\Delta$ alone (NT252) compared with plants containing L3 $\Delta+$ PAP (NT245). L3 mRNA expression level in plants containing the full-length L3 alone (NT250) was two- to fivefold higher than in plants containing the full-length L3+PAP (NT243). As shown in Figure $3 \mathrm{~B}$, analysis of PAP mRNA levels indicated that PAP mRNA was expressed at 10- to 14-fold higher levels in the transgenic lines containing L3 $\Delta+$ PAP (NT245), compared with the line
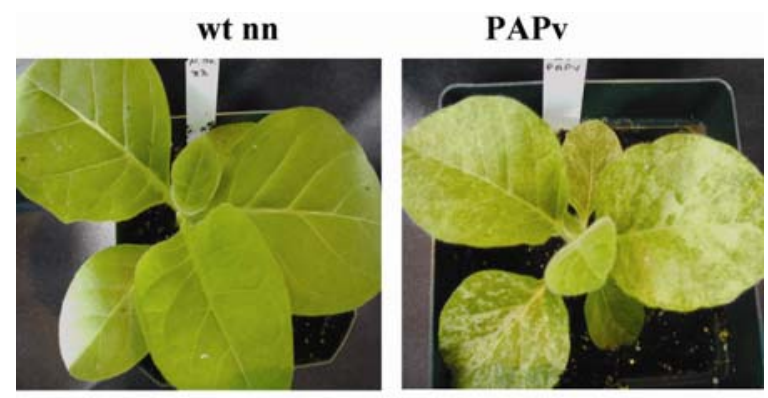

243-6 (L3+PAP)

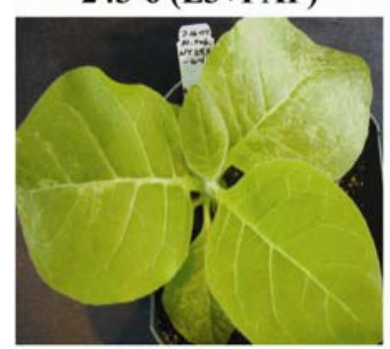

243-8 (L3+PAP)

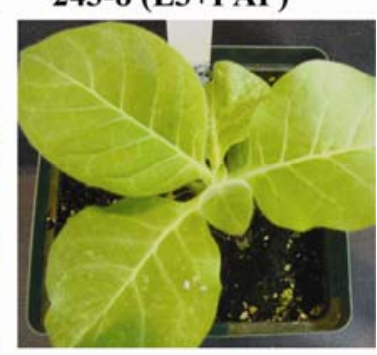

245-1 (L3 $\Delta+$ PAP)

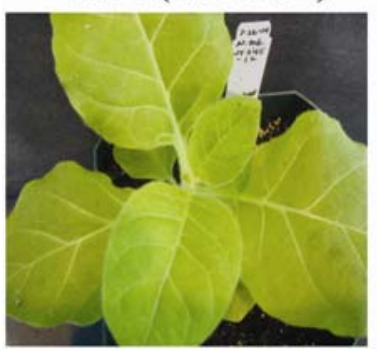

245-2 (L3 $\Delta+$ PAP)

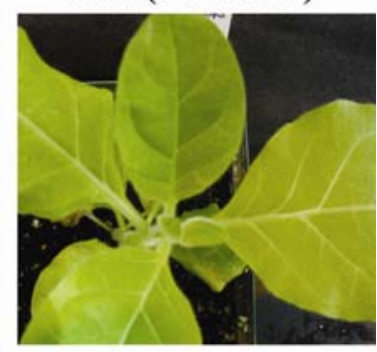

Fig. 2. Comparison of transgenic tobacco plants with wild-type plants. One representative plant from the homozygous progeny (T2 generation) of each transgenic line and a wild-type control plant were photographed 4 weeks after germination. Plants from lines 243-6 and 243-8, containing L3+PAP (pokeweed antiviral protein), showed milder lesions compared with those of plants containing the active PAP (PAPv). In contrast, plants from lines 245-1 and 245-2, containing L3 $\Delta+$ PAP, did not show any lesions on their leaves and were indistinguishable from the wild-type control plants (wt nn). expressing PAPv. PAP mRNA was expressed at twofold higher levels in line 243-8, containing the full-length L3+PAP, compared with the PAPv line (Fig. 3B). These results demonstrated that coexpression of yeast L3 or L3 $\Delta$ led to an increase in accumulation of the PAP mRNA and a decrease in accumulation of the L3 or L3 $\triangle$ mRNA, respectively.

\section{Ribosome depurination in transgenic plants.}

To determine if inhibition of ribosome depurination led to the reduction of cytotoxicity observed in the transgenic lines containing NT243 and NT245, primer extension analysis was used to assess the level of rRNA depurination in the homozygous progeny of these lines. To quantify the extent of depurination in vivo, we used a previously described dual primer extension assay (Parikh et al. 2002), which employs two different primers. One primer that hybridizes downstream of the depurination site was used to examine the extent of depurination of $28 \mathrm{~S}$ rRNA. Another primer that hybridizes upstream of the depurination site (rRNA) was used to quantify the total amount of tobacco 28S rRNA (Fig. 4A). The ratio of the depurination fragment compared with the control fragment allowed for accurate quantification of the extent of depurination. As shown in Figure 4A, rRNA from the homozygous progeny containing L3+PAP (NT243) was depurinated by the constitutively expressed wild-type PAP. However, the level of depurination observed in lines 243-6 and 243-8 was 65 and $60 \%$ of the level of depurination in plants expressing the PAPv, respectively, and did not affect the viability of the seeds.

Ribosomes from the homozygous progeny containing L3 $\Delta+$ PAP (NT245) were not depurinated in vivo (Fig. 4A). However, when the ribosomes from line 245-1 were isolated and treated with purified PAP in vitro, the rRNA was depurinated (Fig. 4A, 245-1+PAP). Similar results were obtained when ribosomes from a wild-type tobacco plant (nn) were isolated and treated with PAP in vitro (wt nn+PAP). Ribosomes were not depurinated in the transgenic lines containing L3 $\Delta+$ PAP, even though these lines expressed much higher levels of PAP than expressed by 243-6 and 243-8, which contained L3+PAP. These results demonstrated that expression of the full-length yeast L3 reduced ribosome depurination, while expression of L3 $\Delta$ prevented ribosome depurination by PAP in vivo.

We have previously shown that PAP is associated with ribosomes in yeast (Hudak et al. 1999) and in transgenic plants (Zoubenko et al. 2000). Since ribosomes were not depurinated in plants containing L3 $\Delta+\mathrm{PAP}$, PAP may not bind to ribosomes. To determine if PAP is associated with ribosomes in plants containing $\mathrm{L} 3+\mathrm{PAP}$ and $\mathrm{L} 3 \Delta+\mathrm{PAP}$, ribosomes were isolated and probed with polyclonal antibody against PAP, followed by polyclonal antibody against tobacco L3. As shown in Figure $4 \mathrm{~B}$, immunoblot analysis indicated that PAP was associated with ribosomes in the transgenic lines containing L3 $\Delta+$ PAP. A lower level of PAP was associated with ribosomes from line 243-8, containing L3+PAP, and PAP was not detected on ribosomes from line 243-6, possibly because this line expressed the lowest levels of PAP (Fig. 1B). These results demonstrated that PAP expressed in transgenic lines containing PAP+L3 $\Delta \Delta$ was associated with ribosomes.

\section{Expression of the endogenous tobacco RPL3 genes.}

To determine if yeast L3 or L3 $\Delta$ affected expression of the endogenous RPL3 genes in tobacco, we analyzed the L3 mRNA and protein levels in the homozygous progeny of transgenic tobacco plants. Real-time PCR analysis was carried out with primers specific for the two tobacco RPL3 genes, which do not cross-hybridize to the yeast RPL3 gene (Popescu and Tumer 2004). The tobacco RPL3A mRNA levels were normalized against the tobacco tubulin A1 mRNA levels as the inter- 
nal control. Real-time PCR analysis was performed at least three times, using two to three replicates per sample. As shown in Figure 5A, the real-time quantitative PCR analysis indicated that the steady state levels of RPL3A mRNA were higher in all the transgenic lines relative to the wild-type tobacco plants (NN or nn) or plants expressing the inactive PAP (PAPx). Plants expressing PAPv displayed the highest level of increase in RPL3A gene expression, with more than a fourfold increase compared with the wild-type plants. Similarly, $R P L 3 B$ levels were higher in all transgenic lines compared with the wildtype plants or the PAPx line (data not shown).

Immunoblot analysis using polyclonal antibodies against tobacco L3, which do not cross-react with the yeast L3 (Popescu and Tumer 2004), indicated that the endogenous L3 protein level was higher in all transgenic lines containing the fulllength yeast L3 or L3 $\Delta$ as well as in the PAPv line compared with the wild-type plants or the PAPx line (Fig. 5B). The PAP protein level was also higher in the transgenic lines containing $\mathrm{L} 3+\mathrm{PAP}$ or L3 $\Delta+\mathrm{PAP}$ compared with the PAPv line (Fig. 5B). PAP accumulation was much higher in the transgenic lines that contained L3 $\Delta+$ PAP compared with the lines that contained L3+PAP (Fig. 5B). The gel was stripped and reprobed with antibodies against phosphoenolpyruvate carboxylase to show equal loading. These results demonstrated that expression of yeast L3 or L3 $\triangle$ up-regulated expression of the endogenous $R P L 3$ genes in the transgenic tobacco plants.
Fungal toxin resistance assay.

To determine if transgenic lines that accumulated higher levels of L3 and PAP are resistant to the Fusarium mycotoxin DON, transgenic and wild-type tobacco seeds were germinated on Murashige-Skoog (MS) medium containing different concentrations of DON. A concentration of $10 \mu \mathrm{M}$ DON was identified as the concentration that would give the best inhibition of wild-type seed growth (data not shown). Four weeks after transgenic tobacco seeds were plated on MS medium containing $10 \mu \mathrm{M}$ DON, the leaves of wild-type plants (nn) became chlorotic (Fig. 6A). In contrast, the leaves of transgenic plants containing L3 $\Delta+\mathrm{PAP}(245-1)$ stayed green (Fig. $6 \mathrm{~A})$. The roots of wild-type plants stopped growing on plates containing $10 \mu \mathrm{M}$ DON (Fig. 6B). In contrast, roots of transgenic plants continued to grow (Fig. 6B). The resistance level was measured by the average root length of 10 plants compared with that of wild-type plants. Plants containing L3 $\Delta+$ PAP showed the highest level of root growth in the presence of DON, almost as much as fourfold compared with the wildtype (NN or $\mathrm{nn}$ ) plants (Fig. 6C). These plants continued to grow in soil and did not show any detrimental effects of DON. Three additional lines, containing L3 $\Delta+$ PAP (245-4, 245-5, and 245-6) germinated on DON-containing MS medium, also displayed resistance to DON (data not shown). Transgenic tobacco lines containing L3+PAP, L3, L3 $\Delta$, and the PAPv line, showed a similar level of root growth in the presence of DON,
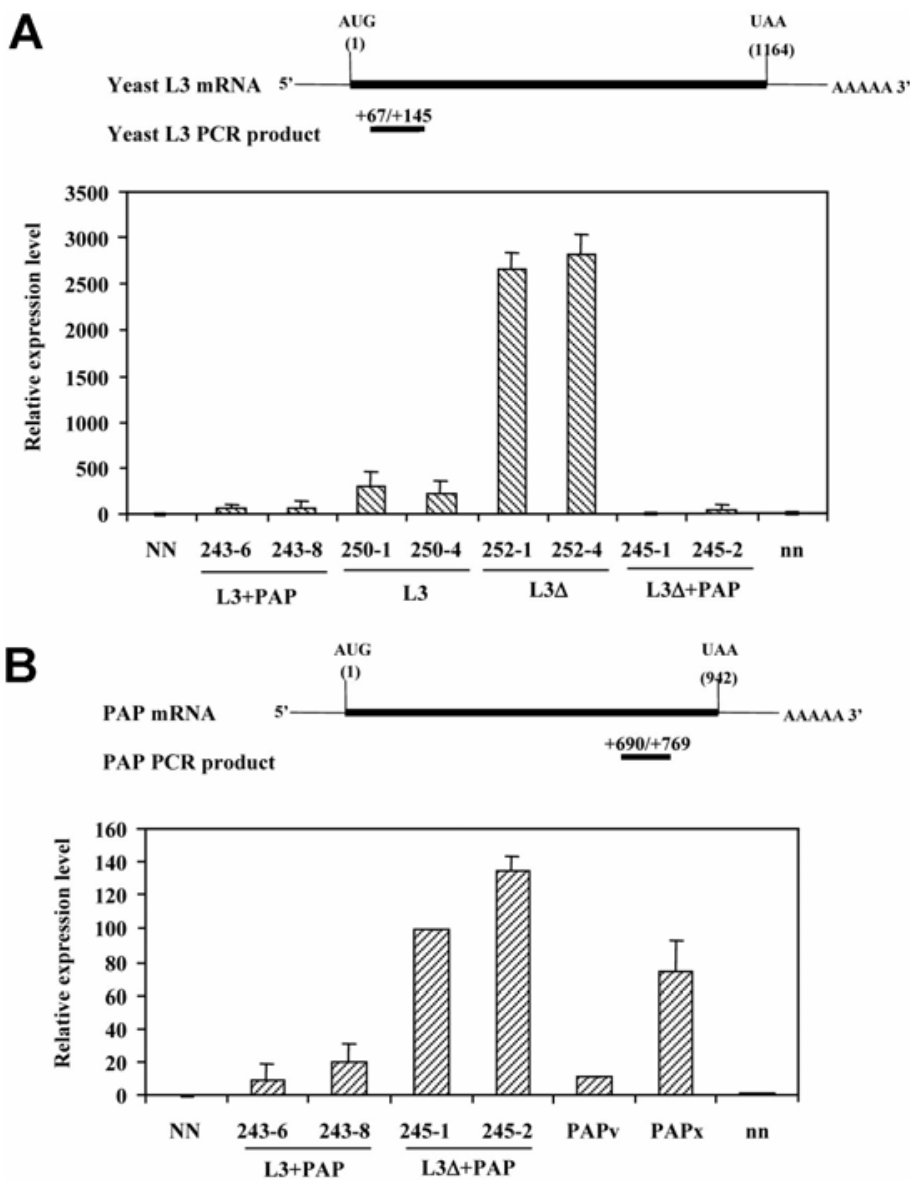

Fig. 3. Steady-state levels of yeast L3 and pokeweed antiviral protein (PAP) mRNA in the transgenic lines. A, Real-time quantitative polymerase chain reaction (PCR) analysis was performed using total RNA from the homozygous progeny of the transgenic lines and primers specific for yeast RPL3. The expression level was normalized against tobacco tubulin A1 mRNA as an internal control. Schematic representation of the yeast RPL3 mRNA, indicating the position the primers used in real-time PCR analysis $(+67 /+145)$, is shown at the top. B, Real-time PCR analysis was performed using total RNA from the homozygous progeny of the transgenic lines and primers specific for PAP. The expression level was normalized against tobacco tubulin A1 mRNA as an internal control. Schematic representation of the PAP mRNA, indicating the position the primers used in real-time PCR analysis (+690/+769), is shown at the top. 
which was lower than the root growth observed in lines containing PAP+L3 $\Delta$ (Fig. 6C). These results demonstrated that coexpression of yeast L $3 \Delta$ and PAP conferred the highest level of resistance to DON.

\section{DISCUSSION}

In recent years, epidemics of FHB or scab have caused devastating yield losses in wheat and barley in the United States (Nganje et al. 2001; Windels 2000). FHB not only causes yield and quality losses but may also result in contamination of all small grain crops, including wheat, barley, oats, and corn, with the mycotoxin, DON (Feinberg and McLaughlin 1989; Khachatourians 1990; McLaughlin et al. 1977). DON causes cell necrosis, smooth muscle paralysis, lower weight gain, and vomiting in animals and, hence, is commonly called vomitoxin. The U. S. Food and Drug Administration (FDA) has recommended limits on DON levels in human food. Effective control measures are needed to control FHB disease in cereals and to prevent trichothecenes, especially DON, from entering the human and animal food chains. Commercially acceptable FHBresistant cultivars have not yet been developed, and the control of FHB by fungicides is ineffective. The first reported plant resistance was with the spring wheat cultivar Frontana, which is resistant to the protein synthesis inhibitory effects of DON (Miller and Ewen 1997). The DON resistance in Frontana and several other cultivars correlates with their resistance to FHB, providing evidence that resistance to DON is a crucial component of resistance to FHB (Miller and Ewen 1997). This is further supported by the observation that gene disruption mutants of Fusarium graminearum that have lost the ability to produce trichothecenes are less virulent on their hosts (McCormick 2003).

Previous attempts to obtain resistance to DON were not very successful. Transgenic tobacco calli and protoplasts carrying a modified rice RPL3 cDNA, containing the W255C mutation found in $t c m-1$, showed greater regeneration efficiency and viability in the presence of DON (Harris and Gleddie 2001). However, sensitivity of the regenerated transgenic plants to DON was not reported in this study (Harris and Gleddie 2001). Expression of a tomato RPL3 cDNA, into which the $t \mathrm{~cm}-1 \mathrm{mu}-$ tation was engineered, improved the ability of transgenic tobacco plants to adapt to DON but did not result in constitutive resistance, due to the lack of accumulation of the mutant protein in the transgenic plants (Mitterbauer et al. 2004).

Other approaches to engineer trichothecene resistance included expression of detoxifying enzymes in transgenic plants. Tobacco plants transformed with either the Saccharomyces cerevisiae PDR5 gene, which encodes a multidrug transporter, or with the Fusarium sporotrichioides TRI101 gene, which encodes a trichothecene 3-O-acetyltransferase, showed increased tolerance to the trichothecene 4,15-diacetoxyscirpenol (Muhitch et al. 2000). Accumulation of TRI101-encoded acetyltransferase in a transgenic wheat plant led to partial protection against the spread of $F$. graminearum (Okubara et al. 2002). Only one line showed moderate level of protection due to the low level of expression of the TRI101 gene in the transgenic wheat plants (Okubara et al. 2002). Transgenic Arabidopsis plants constitutively overexpressing a deoxynivalenol-glucosyl-transferase (DOGT1) gene from Arabidopsis displayed enhanced tolerance against DON (Poppenberger et al. 2003). DOGT1 was shown to detoxify DON by catalyzing the transfer of glucose from UDP-glucose to the $3-\mathrm{OH}$ position of DON, producing $3-\beta-\mathrm{D}-$ glucopyranosyl-4-deoxynivalenol with reduced toxicity. Arabidopsis lines overexpressing DOGT1 showed a delay in development, which is attributed to the reduced toxicity of the detoxified product of DON (Poppenberger et al. 2003).

We regenerated 12 transgenic lines containing the fulllength yeast L3 and PAP with a transformation frequency of $24 \%$, and the majority of plants appeared normal, in stark contrast to our previously reported data in which the transformation frequency with wild-type PAP (pMON8443) was only

A

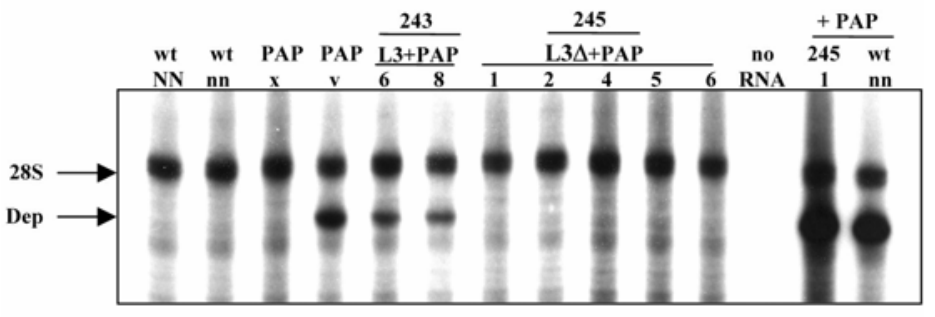

B

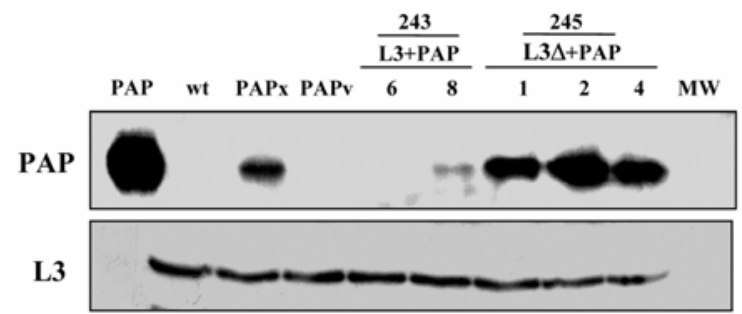

Fig. 4. Analysis of ribosome depurination and association of pokeweed antiviral protein (PAP) with ribosomes in the transgenic lines. A, Primer extension analysis was carried out with total rRNA from the homozygous progeny of lines containing L3+PAP (NT243) and L3 $\Delta+$ PAP (NT245). An end-labeled oligonucleotide primer downstream of the depurination site (Dep) was used to measure the extent of depurination, and another end-labeled oligonucleotide primer upstream of the depurination site was used to measure the total amount of $28 \mathrm{~S}$ rRNA used in the reaction. The extent of depurination was calculated from the ratio of the extension product with the depurination primer to the extension product with the $28 \mathrm{~S}$ rRNA primer. The total RNA isolated from line 245-1 and wild-type plants was treated with purified PAP in vitro (+PAP). B, Ribosomes (10 $\mu \mathrm{g})$ isolated from each line were subjected to immunoblot analysis using polyclonal immunoglobulin G against PAP (1:2,000). The membrane was stripped with $8 \mathrm{M}$ guanidine hydrochloride and reprobed with polyclonal antiserum against tobacco L3 (1:2,000). Purified PAP (50 ng) was used as the standard (PAP). The numbers above each lane refer to the numbers of the transgenic lines. 
$0.7 \%$ and the transgenic plants showed growth defects (Lodge et al. 1993). These results suggested a possible interaction between L3 and PAP in transgenic tobacco plants that led to a reduction of the cytotoxicity of PAP. Several transgenic lines expressing the full-length L3 and wild-type PAP showed lesions that were milder than those observed on plants expressing the active PAP (Fig. 2). Ribosomes were depurinated in the transgenic lines containing the full-length L3 and PAP. However, the level of depurination was only 60 to $65 \%$ of the level observed in plants expressing active PAP, which correlated well with the reduced severity of the lesions.

The reduction in toxicity observed was even greater when the truncated L3 gene, containing only the N-terminal 99 amino acids of L3, was coexpressed with PAP. Transgenic plants expressing L3 $\Delta$ and PAP were phenotypically normal and indistinguishable from wild-type plants. These plants did not display any lesions on their leaves. Ribosome depurination was not detected in any of the lines containing L $3 \Delta$ and PAP even though they expressed high levels of PAP, which was associated with ribosomes. These results suggested that coexpression of L $3 \Delta$ led to inactivation of PAP in transgenic plants.

Since PAP mRNA accumulated to high levels in plants containing L3 $\Delta$, PAP was not able to destabilize its own mRNA in the presence of L3 $\Delta$. In contrast, L3 $\Delta$ mRNA levels were greatly reduced when $\mathrm{L} 3 \Delta$ was coexpressed with PAP. These results suggested that PAP destabilized the yeast L3 $\Delta$ mRNA instead of its own mRNA in the transgenic tobacco plants. The full-length L3 had a similar effect on PAP expression but to a reduced level.
In previous studies, we uncovered the dual ability of PAP to target not only the ribosomes but mRNA as well and showed that PAP binds to the $\mathrm{m}^{7} \mathrm{GpppX}$ cap structure and depurinates the mRNA downstream of the cap in vitro (Hudak et al. 2002). The affinity of PAP for cap was only fourfold lower than its affinity for the SRL (Hudak et al. 2002). The similar affinity of PAP for either the mRNA or the SRL suggested that PAP targets either the rRNA or mRNA but not both. Biochemical analysis confirmed that PAP has a single binding site (Hudak et al. 2002). In vivo evidence for the activity of PAP on mRNA was obtained by demonstrating that PAP destabilizes its own mRNA in yeast (Parikh et al. 2002) and in transgenic plants (U. Baykal and N. E. Tumer, unpublished data). We recently showed that the activity of PAP on mRNA could be dissociated from its activity on the rRNA (Parikh et al. 2005).

We envision two models to explain how the full-length L3 or L3 $\Delta$ may affect PAP mRNA accumulation in transgenic plants. Since, in transgenic plants containing L3 $\Delta$ (NT252), the L3 $\Delta$ mRNA levels are very high, L3 $\Delta$ mRNA, instead of PAP mRNA, may be targeted by PAP. This is further supported by the observation that the $5^{\prime}$ end of L3 mRNA contains a stem-loop structure highly similar to the SRL of the large rRNA. The sequence "AGUACGA" in the L3 mRNA stem loop is identical to the sequence of the SRL "AGUACGAG AGGA," which is the longest conserved sequence found in all large rRNAs (Szewczak and Moore 1995). This sequence is also highly similar to the "GUACGU" sequence in the yeast $R P S 14 B$ mRNA, which is responsible for regulation of expression of the RPS14B gene encoding $\mathrm{S} 14$ (Li et al. 1995). This

\section{A}
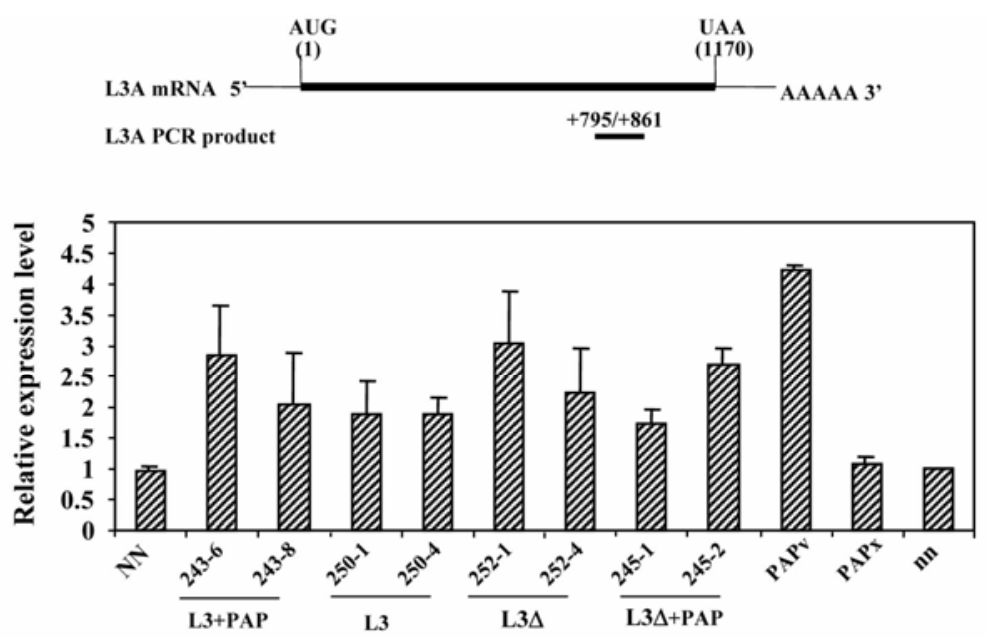

B

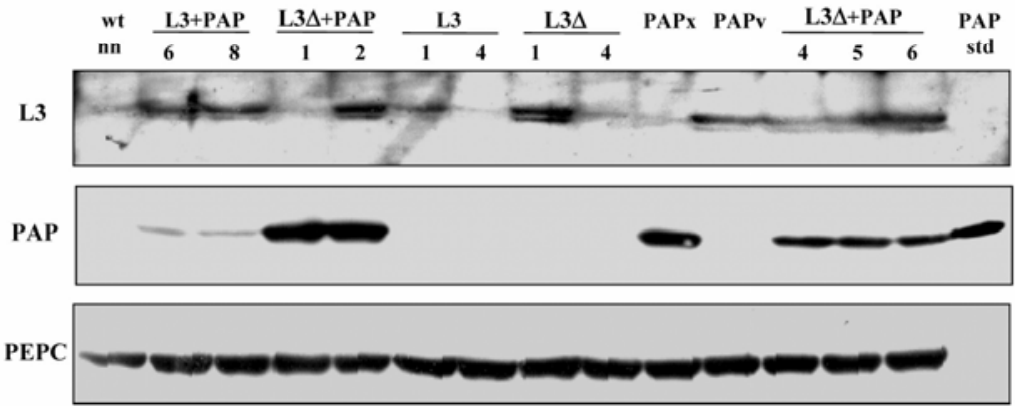

Fig. 5. Analysis of endogenous tobacco RPL3 expression. A, Steady-state levels of mRNA from tobacco RPL3A were analyzed by real-time quantitative polymerase chain reaction (PCR) in the homozygous progeny of transgenic lines. The mRNA levels were normalized against the tobacco tubulin A1 mRNA as an internal control. Schematic representation of the tobacco RPL3 mRNA, indicating the position the primers used in real-time PCR analysis (+795/+861) is shown at the top. B, Immunoblot analysis of endogenous L3 levels in transgenic tobacco plants. Total protein ( $10 \mu \mathrm{g})$ from each sample was subjected to immunoblot analysis using polyclonal antibody against tobacco RPL3 $(1: 2,000)$. The membrane was stripped and reprobed with polyclonal immunoglobulin G against pokeweed antiviral protein (PAP) (1:2,000). The membrane was stripped again and probed with polyclonal antibody against maize phosphoenolpyruvate carboxylase (1:10,000) (PEPC). The numbers above each lane refer to the numbers of the transgenic lines. 
stem loop sequence at the $5^{\prime}$ end of the yeast L3 mRNA may normally regulate $\mathrm{L} 3$ expression by sequestering the L 3 protein, such that binding of L3 to this sequence prevents translation of the L3 mRNA. This is consistent with the observation that L3 cannot be overexpressed in yeast (Pearson et al. 1982). If this sequence acts as an SRL mimic, PAP may bind to it and destabilize the L3 $\Delta$ mRNA instead of its own mRNA. As a result, PAP cannot autoregulate its own expression or depurinate the SRL. This would explain why expression of the yeast L3 or L3 $\Delta$ prevents ribosome depurination and autoregulation of PAP expression. The L $3 \Delta$ may be more effective than the fulllength L3 because it is expressed at higher levels. Furthermore, since the L $3 \Delta$ sequence is conserved between the yeast and tobacco genes, tobacco L3 may bind to this sequence instead of to its own mRNA, up-regulating the endogenous tobacco RPL3 mRNA and protein levels in transgenic plants. Higher levels of endogenous L3 together with higher levels of PAP, which is associated with ribosomes in plants expressing L3 $\Delta+\mathrm{PAP}$, may protect ribosome-bound L3 from the translation inhibitory effects of DON.

The second model is that the observed effect is due to the L3 $\Delta$ peptide. It has long been known that yeast cells harboring the tcm- 1 allele of RPL3 are resistant to trichodermin (FernandezLobato et al. 1990; Fried and Warner 1981; Grant et al 1976; Jimenez et al. 1975; Schindler et al. 1974). An additional allele of RPL3, named mak8-1, was also characterized based on its inability to propagate the killer virus (Wickner et al. 1982). Subsequent results demonstrated that an increase in -1 frameshifting led to the loss of the killer virus in the mak8-1 cells (Peltz et al. 1999). The $t c m-1$ allele of L 3 that conferred resistance to trichodermin also increased -1 frameshifting, reducing the translational fidelity in yeast (Peltz et al. 1999). Furthermore, episomal expression of an L3 $\Delta$ allele had a trans-dominant effect on promoting the programmed -1 ribosomal frameshifting of the killer virus, reducing the translation fidelity in yeast (Peltz et al. 1999). The reduction in translational fidelity in the $t c m-1$ and mak8-1 cells is attributed to a reduction in peptidyltransferase activity (Meskauskas et al. 2003; Petrov et al. 2004).

Previous studies showed that a double-stranded RNA construct corresponding to the $5^{\prime}$ region of the tobacco RPL3A gene up-regulated the L3 protein levels and ribosome biogenesis in transgenic plants (Popescu and Tumer 2004). We show here that endogenous L3 levels are increased in the transgenic tobacco plants, suggesting that ribosome biogenesis may be up-regulated in the presence of the yeast L3 $\Delta$. Upregulation of endogenous L3 levels and ribosome biogenesis may result in incorporation of suboptimal levels of L3 into ribosomes, resulting in a reduction in peptidyltransferase activity. Since PAP targets actively translating ribosomes, ribosomes with reduced peptidyltransferase activity may be less sensitive to depurination by PAP in vivo. Similarly, since DON inhibits peptidyltransferase activity, ribosomes with reduced peptidyltransferase activity may be more resistant to DON. In future studies, we will determine if the resistance to DON reported here will lead to resistance to Fusarium spp. in transgenic wheat plants, since tobacco is not susceptible to infection by $F$. graminearum.

Previous studies demonstrated that expression of PAP in transgenic plants confers broad spectrum resistance to viral infection (Parikh and Tumer 2004; Park et al. 2004; Stirpe 2004). We examined the antiviral activity of PAP in transgenic lines expressing L3 and PAP. Transgenic plants from lines 243-6 and 243-8 expressing L3+PAP showed resistance to Tobacco mosaic virus infection (data not shown). In contrast, we did not observe resistance to Potato virus $X$ infection in lines expressing L $3 \Delta+\mathrm{PAP}$. These results indicate that coexpression of L3 $\Delta$ leads to loss of enzymatic activity of PAP on rRNA, mRNA, and viral RNA, suggesting that L3 $\Delta$ may be an effective inhibitor that can protect cells against PAP.
A

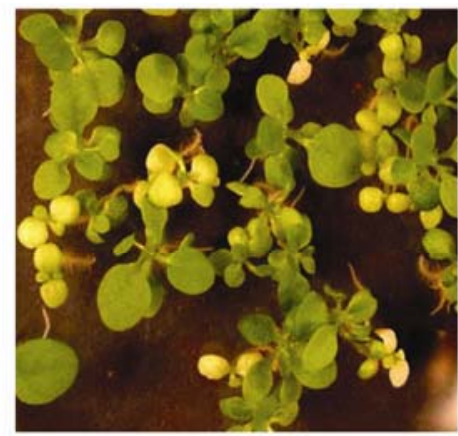

Wild type (nn)

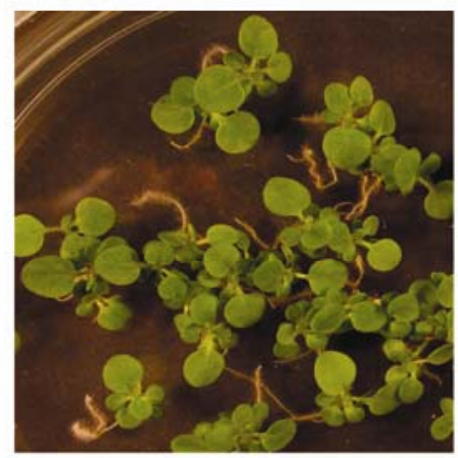

C

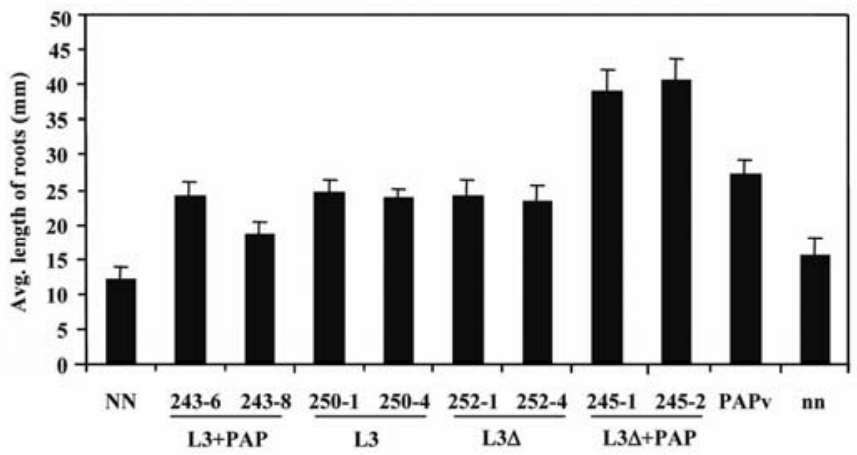

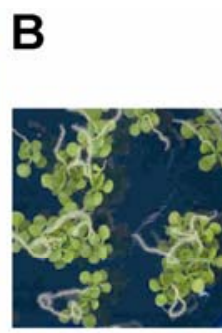

Wt (nn)

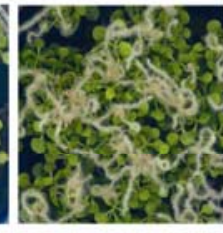

243-8

243-8

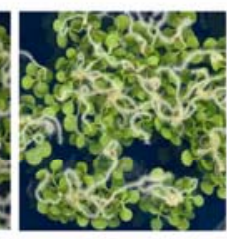

250-4

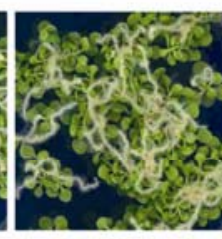

252-4

245-1 (L3 $\Delta+$ PAP)

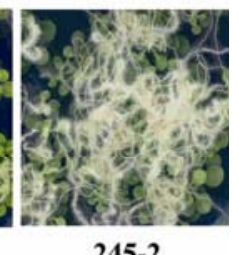

245-2

Fig. 6. Analysis of resistance to deoxynivalenol (DON). Tobacco seeds were surface-sterilized and germinated on Murashige-Skoog medium containing 10 $\mu \mathrm{M}$ DON. A, Comparison of the leaves of wild-type and transgenic plants 4 weeks after germination on DON. B, Comparison of the roots of wild-type and transgenic plants 4 weeks after germination on DON. C, The root length of 10 plants from each construct was measured and averaged 4 weeks after germination on DON. 
In summary, the results of this study demonstrate that overexpression of the $\mathrm{N}$-terminal 99 amino acids of ribosomal protein L3 confers trans-dominant resistance to PAP and the trichothecene mycotoxin DON, providing evidence that both toxins target L3 by a common mechanism. These results demonstrate that L3 presents a novel target for controlling DON accumulation in small grain cereals and for developing novel technologies that can counteract the potential use of RIPs as agents of bioterror.

\section{MATERIALS AND METHODS}

\section{Plant expression vectors.}

Wild-type L3 gene and L3 $\Delta$ (the N-terminal 99 amino acid of L3) from yeast were cloned downstream of the enhanced $35 \mathrm{~S}$ promoter from Cauliflower mosaic virus, resulting in NT250 and NT252, respectively. Yeast L3 and L3 $\Delta$ were cloned into the vector containing PAP to generate NT243 and NT245, respectively. NPTII served as the selection marker. All constructs were transformed into tobacco, Nicotiana tabacum cv. Samsun NN or nn using Agrobacterium-mediated transformation, as previously described (Tumer et al. 1997). Enzyme-linked immunosorbent assay analysis for NPTII expression (Agdia, Elkhart, IN, U.S.A.) was performed to identify transgenic plants. Presence of the yeast L3 and PAP genes was confirmed by PCR analysis. Homozygous progeny of transgenic lines (T2 generation) were used in the experiments described below.

\section{Ribosome isolation and analysis of rRNA depurination.}

Ribosomes were isolated from transgenic plants as previously described (Zoubenko et al. 2000). The rRNA extracted from the ribosomes was analyzed by a dual primer extension assay, as described previously (Parikh et al. 2002). Extension products were separated on a $7 \mathrm{M}$ urea, $5 \%$ polyacrylamide gel and were quantified using a Phosphorimager (Molecular Dynamics, Sunnyvale, CA, U.S.A.).

\section{Fungal toxin resistance assay.}

To determine the resistance of transgenic tobacco plants to fungal toxins, a sensitive seed-germination assay was developed. MS medium (Invitrogen, Carlsbad, CA, U.S.A.) with 30 $\mathrm{g}$ of sucrose per liter containing DON was solidified in petri dishes with agar. Seeds from the homozygous progeny of each transgenic line were surface-sterilized with $50 \%$ bleach containing $0.1 \%$ Triton $\mathrm{X}-100$ for $10 \mathrm{~min}$, were rinsed with sterile water three times, and were distributed onto the petri dishes with MS medium. Dishes were incubated under 16-h light and 8 -h dark cycle at $25^{\circ} \mathrm{C}$. The germination of the seeds and the growth of the seedlings were recorded, and the root length was measured after 4 weeks of growth on DON.

\section{Real-time quantitative PCR.}

Total RNA was isolated from the leaves of transgenic tobacco plants using TRIzol Reagent (Invitrogen) according to the manufacturer's protocol. SuperScript reverse transcriptase (Invitrogen) and oligo dT primers were used to produce firststrand cDNA from $5 \mu \mathrm{g}$ of total RNA. Real-time PCR analysis was performed, using gene-specific primers with ABI PRISM 7000 Sequence Detection System (Applied Biosystems, Foster City, CA, U.S.A.), using the manufacturer's protocols. For PAP gene, the primers used were PAP690F, 5'-GGGTAAGA TTTCAACAGCAATTCA-3' and PAP769R, 5'-CACCACTGG CATCCACTAGCT-3'. The primers for yeast L3 and L3 $\Delta$ genes were L3d67F, 5'-GCCTCCATCAGAGCTAGAGTTAA GG-3' and L3d145R, 5'-AACCCAAGAAGGAAGTTAGAGC AA-3'. The primers for tobacco L3A gene were L3A795F, 5'GGTCTGCGTAAGGTTGCTTGT-3' and L3A861R, 5'-CACG
GGCAACTGTGTAGGAA-3'. The primers used to detect tobacco L3B gene were L3B842F, 5'-AACCCGTCTCCCACG TAAGA-3' and L3B907R, 5'-GGATGCCAAGCACCAATAC A-3'. Transcript levels were normalized against tobacco tubulin A1 mRNA as an internal control using the ddCT method (Livak and Schmittgen 2001).

\section{ACKNOWLEDGMENTS}

The authors would like to thank J. Dinman, for the gift of yeast L3 plasmid and helpful discussions, E. Lam, M. Lawton, and X.-P. Li for critically reading the manuscript. This work was supported by the United States Department of Agriculture-Agricultural Research Service to N. E. Tumer under agreement number 58-5325-2-758 and the National Science Foundation (MCB 0130531 and MCB 0348299 to N. E. Tumer). This is a cooperative project with the U.S. Wheat and Barley Scab Initiative.

\section{LITERATURE CITED}

Bolognesi, A., and Polito, L. 2004. Immunotoxins and other conjugates: Pre-clinical studies. Mini-Rev. Med. Chem. 4:563-583.

Christopher, G. W., Cieslak, T. J. Pavlin, J. A., and Eitzen, E. M., Jr. 1997. Biological warfare. A historical perspective. J Amer. Med. Assoc. 278:412-417.

Feinberg, B., and McLaughlin, C. S. 1989. Biochemical mechanism of actions of trichothecene mycotoxins. Pages 27-35 in: Trichothecene Mycotoxicosis: Pathophysiologic Effects. V. R. Beasley, ed. CRC Press, Boca Raton, FL, U.S.A.

Fernandez-Lobato, M., Cannon, M., Mitlin, J. A., Mount, R. C., and Jimenez, A. 1990. Characterization of Saccharomyces cerevisiae strains displaying high-level or low-level resistance to trichothecene antibiotics. Biochem. J. 267:709-713.

Fried, H. M., and Warner, J. R. 1981. Cloning of yeast gene for trichodermin resistance and ribosomal protein L3. Proc. Natl. Acad. Sci. U.S.A. 78:238-242.

Grant, P. E., Schindler, D., and Davies, J. E. 1976. Mapping of trichodermin resistance in Saccharomyces cerevisae: A genetic locus for a component of the 60S ribosomal subunit. Genetics 83:667-673.

Hampl, H., Schulze, H., and Nierhaus, K. H. 1981. Ribosomal components from Escherichia coli 50S subunits involved in the reconstitution of peptidyltransferase activity. J. Biol. Chem. 256:2284-2288.

Harris, L. J., and Gleddie, S. C. 2001. A modified Rpl3 gene from rice confers tolerance of the Fusarium graminearum mycotoxin deoxynivalenol to transgenic tobacco. Physiol. Mol. Pl. Path. 58:173-181.

Henghold, W. B., II. 2004. Other biologic toxin bioweapons: Ricin, staphylococcal enterotoxin B and trichothecene mycotoxins. Dermatol. Clin. 22:257-262.

Hudak, K. A., Bauman, J. D., and Tumer, N. E. 2002. Pokeweed antiviral protein binds to the cap structure of eukaryotic mRNA and depurinates the mRNA downstream of the cap. RNA 8:1148-1159.

Hudak, K. A., Dinman, J. D., and Tumer, N. E. 1999. Pokeweed antiviral protein accesses ribosomes by binding to L3. J. Biol. Chem. 274:38593864.

Jimenez, A., Sanchez, L., and Vazquez, D. 1975. Simultaneous ribosomal resistance to trichodermin and anisomycin in Saccharomyces cerevisiae mutants. Biochim. Biophys. Acta 383:427-434.

Khachatourians, G. G. 1990. Metabolic effects of trichothecene T-2 toxin. Can. J. Physiol. Pharm. 68:1004-1008.

Li, Z., Paulovich, A. G., and Woolford, J. L. 1995. Feedback inhibition of the yeast ribosomal protein gene $C R Y 2$ is mediated by the nucleotide sequence and secondary structure of $C R Y 2$ pre-mRNA. Mol. Cell. Biol. 15:6454-6464.

Livak, K. J., and Schmittgen, T. D. 2001. Analysis of relative gene expression data using real-time quantitative PCR and the $2^{-\Delta \Lambda \mathrm{CT}}$ method. Methods 25:402-408.

Lodge. J. K., Kaniewski, W. K., and Tumer, N. E. 1993. Broad-spectrum virus resistance in transgenic plants expressing pokeweed antiviral protein. Proc. Natl. Acad. Sci. U.S.A. 90:7089-7093.

McCormick, S. 2003. The role of DON in pathogenicity. Pages 165-183 in: Fusarium Head Blight of Wheat and Barley. K. J. Leonard and W. R. Bushnell, eds. American Phytopathological Society Press, St. Paul, MN, U.S.A.

McLaughlin, C. S., Vaughan, M. H., Campbell, I. M., Wei, C. M., Stafford, M. E., and Hansen, B.S. 1977. Inhibition of protein synthesis by trichothecenes. Pages 262-173 in: Mycotoxins in Human and Animal Health. J. V. Rodricks, C. W. Hesseltine, M. A. Mehlman, eds. Patho Publishers, Inc., Park Forest South, IL, U.S.A. 
McMullen, M., Jones, R., and Gallenberg, D. 1997. Scab of wheat and barley: A re-emerging disease of devastating impact. Plant Dis. 81:1340-1348.

Meskauskas, A., Harger, J. W., Jacobs, K. L. M., and Dinman, J. D. 2003. Decreased peptidyltransferase activity correlates with increased programmed -1 ribosomal frameshifting and viral maintenance defects in the yeast Saccharomyces cerevisiae. RNA 9:982-992.

Miller, J. D., and Ewen, M. A. 1997. Toxic effects of deoxynivalenol on ribosomes and tissues of the spring wheat cultivars Frontana and Casavant. Nat. Toxins 5:234-237.

Mitterbauer, R., Poppenberger, B., Raditschnig, A., Lucyshyn, D., Lemmens, M., Glossl, J., and Adam, G. 2004. Toxin-dependent utilization of engineered ribosomal protein L3 limits trichothecene resistance in transgenic plants. Plant Biotech. J. 2:329-340.

Muhitch, M. J., McCormick, S. P., Alexander, N. J., and Hohn, T. M. 2000. Transgenic expression of the TRI101 or PDR5 gene increases resistance of tobacco to the phytotoxic effects of the trichothecene 4,15-diacetoxyscirpenol. Plant Sci. 157:201-207.

Nganje, W. E., Johnson, D. D., Wilson, W. W., Leistritz, F. L., Bangsund, D. A., and Tiapo, N. M. 2001. Economic impacts of Fusarium head blight in wheat and barley: Agribusiness and applied economics. Report No. 464. Department of Agriculture and Applied Economics, North Dakota State University, Fargo, ND, U.S.A. U.S. Wheat and Barley Scab Initiative. scabusa.org/pdfs/98-00_Economics_Report.PDF. Online publication.

Okubara, P. A., Blechl, A. E., McCormick, S. P., Alexander, N. J., DillMacky, R., and Hohn, T. M. 2002. Engineering deoxynivalenol metabolism in wheat through the expression of a fungal trichothecene acetyltransferase gene. Theor. Appl. Genet. 106:74-83.

Olsnes, S., and Sandvig, K. 1988. How protein toxins enter and kill cells. Pages 39-73 in: Immunotoxins. A. E. Frankel, ed. Kluwer Academic Publishers, Boston.

Parikh, B. A., Baykal, U., Di, R., and Tumer, N. E. 2005. Evidence for retro-translocation of pokeweed antiviral protein from the endoplasmic reticulum into the cytosol and separation of its activity on ribosomes from its activity on capped RNA. Biochemistry 44:2478-2490.

Parikh, B. A., Coetzer, C., and Tumer, N. E. 2002. Pokeweed antiviral protein regulates the stability of its own mRNA by a mechanism that requires depurination but can be separated from depurination of the alpha-sarcin/ricin loop of rRNA. J. Biol. Chem. 277:41428-41437.

Parikh, B. A., and Tumer, N. E. 2004. Antiviral activity of ribosome inactivating proteins in medicine. Mini Rev. in Med. Chem. 4:529-549.

Park, S. W., Vepachedu, R., Sharma N., and Vivanco, J. M. 2004. Ribosome-inactivating proteins in plant biology. Planta 219:1093-1096.

Pearson, N. J., Fried, H. M., and Warner, J. R. 1982. Yeast use translational control to compensate for extra copies of a ribosomal protein gene. Cell 29:347-355.

Peltz, S. W., Hammell, A. B., Cui, Y., Yasenchak, J., Puljanowski, L., and Dinman, J. D. 1999. Ribosomal protein L3 mutants alter translational fidelity and promote rapid loss of the yeast killer virus. Mol. Cell. Biol. 19:384-39.

Petrov, A., Meskauskas, A., and Dinman, J. D. 2004. Ribosomal protein L3. RNA Biol. 1:59-65.

Popescu, S. C., and Tumer, N. E. 2004. Silencing of ribosomal protein L3 genes in $N$. tabacum reveals coordinate expression and significant alterations in plant growth, development and ribosome biogenesis. Plant J. 39:29-44.

Poppenberger, B., Berthiller, F., Lucyshyn, D., Sieberer, T., Schuhmacher R., Krska, R., Kuchler, K., Glossl, J., Luschnig, C., and Adam, G. 2003. Detoxification of the Fusarium mycotoxin deoxynivalenol by a UDPglucosyltransferase from Arabidopsis thaliana. J. Biol. Chem. 278:47905-47914.

Proctor, R. H., Hohn, T. M., and McCormick, S. P. 1995. Reduced virulence of Gibberella zeae caused by disruption of a trichothecene toxin biosynthetic gene. Mol. Plant-Microbe Interact. 8:593-601.

Roth, H. E., and Nierhaus, K. H. 1980. Assembly map of the 50S subunit from Escherichia coli ribosomes, covering the proteins present in the first reconstitution intermediate particle. Eur. J. Biochem. 103:9598.

Schindler, D., Grant, P., and Davies, J. 1974. Trichodermin resistance-mutation affecting eukaryotic ribosomes. Nature 248:535-536.

Stirpe, F. 2004. Ribosome-inactivating proteins. Toxicon 44:371-383.

Szewczak, A. A., and Moore, P. B. 1995. The sarcin/ricin loop, a modular RNA. J. Mol. Biol. 247:81-98.

Tumer, N. E., Hwang, D. J., and Bonness, M. 1997. C-terminal deletion mutant of pokeweed antiviral protein inhibits viral infection but does not depurinate host ribosomes. Proc. Natl. Acad. Sci. U.S.A. 94:38663871

Uchiumi, T., Sato, N., Wada, A., and Hachimori, A. 1999. Interaction of the sarcin/ricin domain of 23S ribosomal RNA with proteins L3 and L6. J. Biol. Chem. 274:681-686.

Wickner, R. B., Porter-Ridley, S., Fried, H. M., and Ball, S. G. 1982. Ribosomal protein L3 is involved in replication or maintenance of the killer double-stranded RNA genome of Saccharomyces cerevisiae. Proc. Natl. Acad. Sci. U.S.A. 79:4706-4708.

Windels, C. E. 2000. Economic and social impacts of Fusarium head blight: Changing farms and rural communities in the Northern Great Plains. Phytopathology 90:17-21.

Wool, I. G., Correll, C. C. and Chen, Y.-L. 2000. Structure and function of the sarcin-ricin domain. Pages 461-473 in: The Ribosome: Structure, Function, Antibiotics, and Cellular Interactions. R. A. Garrett, S. R. Douthwaite, A. Liljas, A. T. Matheson, P. B. Moore, and H. F. Noller, eds. American Society for Microbiology Press, Washington, DC.

Zapor, M., and Fishbain, J. T. 2004. Aerosolized biologic toxins as agents of warfare and terrorism. Resp. Care Clin. N. Am. 10:111-22.

Zoubenko, O., Hudak, K. A., and Tumer, N. E. 2000. A non-toxic pokeweed antiviral protein mutant inhibits pathogen infection via a novel salicylic acid-independent pathway. Plant Mol. Biol. 44:219-229. 